\title{
Component and System Sensitivity Considerations for Design of a Lunar ISRU Oxygen Production Plant
}

\author{
Diane L. Linne, ${ }^{1}$ and Suleyman Gokoglu ${ }^{2}$ \\ NASA Glenn Research Center, Cleveland, OH 44135, USA \\ Uday G. Hegde ${ }^{3}$ and Ramaswamy Balasubramaniam ${ }^{4}$ \\ National Center for Space Exploration, Cleveland, OH 44135, USA \\ and \\ Edgardo Santiago-Maldonado ${ }^{5}$ \\ NASA Kennedy Space Center, FL, USA
}

\begin{abstract}
Component and system sensitivities of some design parameters of ISRU system components are analyzed. The differences between terrestrial and lunar excavation are discussed, and a qualitative comparison of large and small excavators is started. The effect of excavator size on the size of the ISRU plant's regolith hoppers is presented. Optimum operating conditions of both hydrogen and carbothermal reduction reactors are explored using recently developed analytical models. Design parameters such as batch size, conversion fraction, and maximum particle size are considered for a hydrogen reduction reactor while batch size, conversion fraction, number of melt zones, and methane flow rate are considered for a carbothermal reduction reactor. For both reactor types the effect of reactor operation on system energy and regolith delivery requirements is presented.
\end{abstract}

\section{Introduction}

NASA's current exploration vision includes plans to go back to the moon for extended stays to perform scientific research and exploration, and in preparation for future missions to Mars. To enable long duration stays of up to six months, it is imperative that technologies to reduce the required logistics and consumable resupply train from the Earth are developed. A leading candidate for reducing the consumables delivery from Earth is to learn to live off the land by utilizing in-situ resources, such as extracting oxygen from the lunar regolith to provide breathing air for the habitat, airlock use, and EVA suits, and eventually make enough for propulsion for the ascent vehicle or surface mobility vehicles such as hoppers. ${ }^{1-3}$

The concept of in-situ resource utilization (ISRU) has been proposed and studied since before the first moon landing, ${ }^{4}$ and significant research on reactor concepts has been performed. Some research and analysis has also been performed on other system components such as excavators, water electrolyzers, and oxygen liquefaction and storage. However, it has only been with the recent renewed effort toward returning to the moon that the focus has turned toward the entire end-to-end oxygen production system. Earlier designs for the entire plant have generally evaluated a single set of top-level requirements, with no ability to understand the multitude of interactions between components and operational logistics. There also has been no capability to understand the sensitivities of the individual components to improvements in technology.

\footnotetext{
${ }^{1}$ Senior Research Engineer, Power and In-Space Propulsion Division, MS 301-3, AIAA Associate Fellow.

${ }^{2}$ Senior Scientist, Space Processes and Experiments Division, MS 77-5.

${ }^{3}$ Research Associate Professor, National Center for Space Exploration Research Center, NASA GRC/Case Western Reserve University, Cleveland, OH, AIAA Associate Fellow.

${ }^{4}$ Research Associate Professor, National Center for Space Exploration Research Center, NASA GRC/Case Western Reserve University, Cleveland, $\mathrm{OH}$.

${ }^{5}$ Chemical Engineer, Applied Technology Directorate, MS KT-D3.
} 
For the last few years, a multi-center NASA team has been developing such an analysis tool to determine the mass, power, and volume of end-to-end oxygen production systems including regolith excavation and handling, oxygen extraction from regolith, gas and fluid processing subsystems, and oxygen liquefaction and storage. This tool has already enabled the ISRU team to understand where peak power requirements develop, and how the use of parallel reactors, for example, can significantly reduce peak power by splitting up times for reactor fill and dump, regolith heating, and oxygen extraction. ${ }^{5,6}$ Component models that comprise this end-to-end system tool are also being used to aid in the design of prototype hardware and predict expected performance for various anticipated regolith simulants.

\section{General Description of ISRU System}

To understand the component and system sensitivities, the general design of the ISRU system was defined. For the study described in this paper the following assumptions were made:

- The ISRU plant produces $500 \mathrm{~kg}$ of oxygen per year. While the current baseline for a fully operating lunar outpost requires $1000 \mathrm{~kg}$ of oxygen per year from ISRU, the proposed $500 \mathrm{~kg}$ initial plant will a) be easier to package on an early flight, b) will provide sufficient oxygen for the outpost before year-round occupation, c) can provide some redundancy if two plants are used for the $1000 \mathrm{~kg} /$ year requirement, and d) may allow an opportunity to improve on the design for the second and/or larger plants.

- The outpost is located at the lunar south pole, providing near-continuous sunlight (i.e., solar power) for much of the year. The ISRU plant is assumed to operate for 70 percent of the year, which allows it to stay dormant for the two poorest sunlight months and still maintain a 10 percent operating time margin.

- The soil at this polar location is relatively low in ironbearing minerals, assumed to be 5 percent, which affects the potential yield of the hydrogen reduction process. Assuming 100 percent conversion of the oxygen in the iron-bearing minerals, the hydrogen

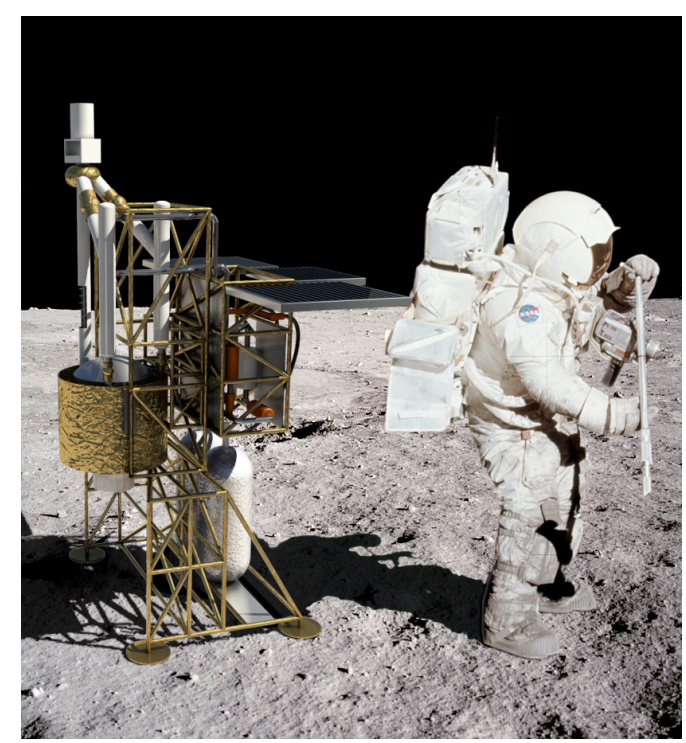

Figure 1. Graphical depiction of early ISRU plant (500 kg oxygen per year). reduction process results in a 1 percent overall yield.

Therefore, a minimum of $50,000 \mathrm{~kg}$ of regolith is required to produce $500 \mathrm{~kg} / \mathrm{yr}$ of oxygen using hydrogen reduction at this site. At 70 percent operations, this is approximately $200 \mathrm{~kg}$ of regolith per 24 -hour day of operation.

- The concentration of silicon, iron, and titanium oxides combined is relatively consistent at both the mare and the polar/highland locations, and the maximum potential oxygen content from these minerals was assumed to be a conservative 25 percent. Assuming a conservative conversion of 40 percent of the oxygen in these minerals, the carbothermal reduction process results in a 10 percent overall yield. Therefore, approximately $5,000 \mathrm{~kg}$ of regolith is required to produce $500 \mathrm{~kg} / \mathrm{yr}$ of oxygen. At 70 percent operations, this is approximately $20 \mathrm{~kg}$ of regolith per 24-hour day of operation.

- Heat recuperation methods are employed to transfer heat from the spent regolith to the cold fresh regolith to reduce the electrical power required to heat up each batch of regolith for the hydrogen reduction. The concept assumed here is for concentric hoppers with the hot spent regolith in the inner hopper and the fresh regolith in the outer hopper. There are many other concepts being considered to capture this energy so this concept was solely used to provide a basis for understanding hopper mass growth.

A graphical depiction of the baseline early ISRU plant described here is shown in Fig. 1. The concentric hoppers are shown on the left side of the plant, and are raised up to allow room for a large excavator to move its scoop underneath the dump hopper to receive spent regolith. An auger rises up from the outer hopper lifting fresh regolith from the hopper into either of the two vertical reactors. On the right side of the plant are the remaining fluid components, radiators, and two liquid oxygen storage tanks. Some items are not drawn for clarity, such as the insulation around the two hydrogen reduction reactors. 


\section{Excavation}

The first step in extracting oxygen from the lunar regolith is to excavate the regolith and deliver it to the production plant. While we have been digging dirt by hand on Earth for thousands of years and for over a hundred years using machinery, there are several ways that excavation on the moon will be different than on Earth. The first set of differences is operational. The relative importance of each factor is summarized in Table I and described below:

- Mass: on Earth large is a cost-effective method of providing sufficient reaction force for digging. On the moon, the mass of all equipment needs to be minimized, as the cost of launching the equipment is significant. In addition, the mass of the ISRU system (including excavation) needs to be significantly less than the mass of the oxygen being produced to maximize the benefits of in-situ production.

- Power: on Earth vehicle power is not a significant concern as there is a relatively inexpensive source of power in the form of consumable fuels for internal combustion engines. On the moon, power must come from a renewable source to avoid the delivery of large

\section{Table I. Design and operations comparison between terrestrial and lunar excavation requirements.}

\begin{tabular}{|l|c|c|}
\cline { 2 - 3 } \multicolumn{1}{c|}{} & \multicolumn{2}{c|}{ Importance Level } \\
\cline { 2 - 3 } \multicolumn{1}{c|}{} & $\begin{array}{c}\text { Terrestrial } \\
\text { Excavation }\end{array}$ & $\begin{array}{c}\text { Lunar } \\
\text { Excavation }\end{array}$ \\
\hline Mass & Low & High \\
\hline Power & Low & High \\
\hline Time & High & Low \\
\hline Autonomy & Low & High \\
\hline Maintenance & Medium & High \\
\hline
\end{tabular}
amounts of consumables to provide excavation power.

- Time: on Earth time is a significant concern as the excavation company earns money for each job completed and therefore time is money. This results in large terrestrial excavation vehicles that can dig large amounts of dirt quickly to complete the job. On the moon the amount of regolith required to produce $1000 \mathrm{~kg}$ of oxygen per year using the low efficiency hydrogen reduction process equates to digging up a coffee cup of dirt every 2 minutes.

- Operation and maintenance: On Earth each excavator has one or more dedicated operators during digging and maintenance procedures. On the moon having astronauts dedicated to excavation operation or frequent maintenance will be cost prohibitive, thus increasing the importance of autonomous operation and long-life and reliable parts.

The second set of differences between lunar and terrestrial excavation is related to the environment:

- Soil: on Earth the soil is highly weathered, varies greatly by location, and often has a high moisture content. On the moon the soil particles are elongated and jagged, very dry, have a high fines fraction in the upper layers, and are relatively homogenous over large areas, with significant differences expected only between the mare and highlands regions. These differences result in different cohesiveness, soil-tool interactions, and flow properties that will affect excavation vehicle/tool designs and performance.

- Atmospheric conditions: the hard vacuum conditions on the moon will affect seals, joint designs, and lubrication. Excavators on the moon will also see more extreme cold temperatures and more frequent extreme thermal cycles.

- Gravity: the lower gravity on the moon means that the same vehicle mass will provide less reaction force for digging into the same soil type. This effect is non-linear and dependent on vehicle type, wheel diameter and width, and soil type and density. ${ }^{7}$

There are currently two options being considered for excavation vehicles. The first option is to attach an excavation tool to a large, multi-purpose mobility vehicle such as the vehicle that will carry the astronauts on pressurized and unpressurized exploration sorties. This vehicle would be designed to meet a wide range of mobility needs, including crewed exploration sorties either in suits or in a pressurized cab, transportation of outpost hardware assets from the lander to the outpost site, uncrewed exploration and prospecting sorties, and local transportation around the outpost. Advantages of a large multi-purpose mobility chassis for excavation include possible reduction in design and development costs and the ability to move larger amounts of regolith faster.

The second option for an excavation vehicle is a smaller vehicle that is designed specifically for excavation and dedicated to supporting the ISRU plant. Because the quantities of regolith required for oxygen production are not that large, one or more small vehicles can easily provide the required regolith for oxygen production. A dedicated vehicle may allow more design flexibility to optimize for excavation if it is not also required to perform a host of other tasks like the large multi-purpose vehicle. Analytical models are being developed that calculate mass and power consumption for different excavation concepts, ${ }^{8}$ but these are not yet sufficiently validated with experimental data to perform a quantitative comparison between small and large excavators in this paper. 
Because the analysis in this paper is focused on producing oxygen from the regolith, we also do not evaluate larger excavation tasks such as clearing landing pads or building berms. For these tasks, a separate evaluation is required that considers dedicating more time from the large, multi-purpose vehicle for excavation tasks compared to using multiple small dedicated excavators.

\section{A. Excavator Type Effects on ISRU Plant}

Large, multipurpose mobility vehicles used for excavation will have many tasks to perform each day, and therefore should accomplish excavation tasks as quickly as possible. At one extreme, the ISRU oxygen plant would receive a batch of regolith only once every few days so that the mobility chassis could spend more time on other activities or even away from the outpost on an exploration sortie. This concept of operation would require large regolith hoppers to hold the fresh regolith where it can be transferred to the reactors, and to hold the spent regolith until it can be carried away with the next visit of the excavator. Figure 2 shows how the mass of concentric regolith hoppers increases as the amount of regolith increases, as measured by the number of days of 'buffer' contained in the hoppers. The hoppers were assumed to have $0.003 \mathrm{~m}$ thick aluminum walls, with a height-to-width ratio of 1.5 for the inner hopper, and 10 percent empty space at the top of each hopper. The advantage of the smaller, dedicated

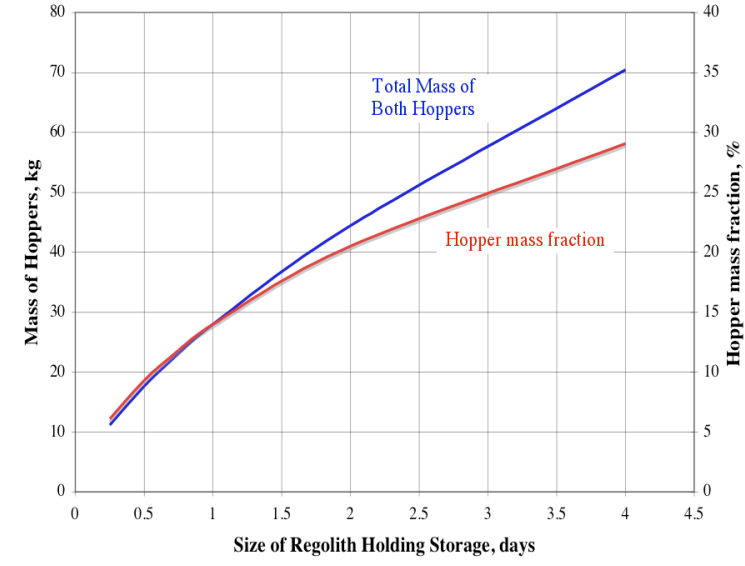

Figure 2. Growth of regolith hopper mass with increasing storage requirement. excavators is that they could deliver several times a day and therefore the hoppers would remain small. Assuming $260 \mathrm{~kg}$ for the total mass of an ISRU system that makes $500 \mathrm{~kg}$ of oxygen per year (excavation implement, hoppers with 1 day of regolith, and oxygen plant), the second curve in Fig. 2 shows how the mass of the hoppers compares to the overall mass of the ISRU system. The hoppers represent six percent of the total mass with four deliveries per day and increase to more than 30 percent of the total mass with one delivery every four days. In addition to the increased mass of the hoppers, the size can also affect the flowability of fresh regolith and processed regolith within the hoppers and the potential for self-compaction with time. These effects have not yet been quantified.

\section{B. Excavator Operations}

Assuming that a large excavator makes a single trip to the ISRU plant each day, the most efficient excavation concept would be to design a single digging scoop to dig up $200 \mathrm{~kg}$ in a single scoop and then drive to the plant and deliver the regolith into the hoppers. There are many digging concepts being evaluated and mass estimates for this large excavation tool or the mechanism to raise it up to dump into the hoppers are not yet completed. In general, a narrower scoop blade will require a deeper or longer cut to gather the $200 \mathrm{~kg}$, which will result in more energy for
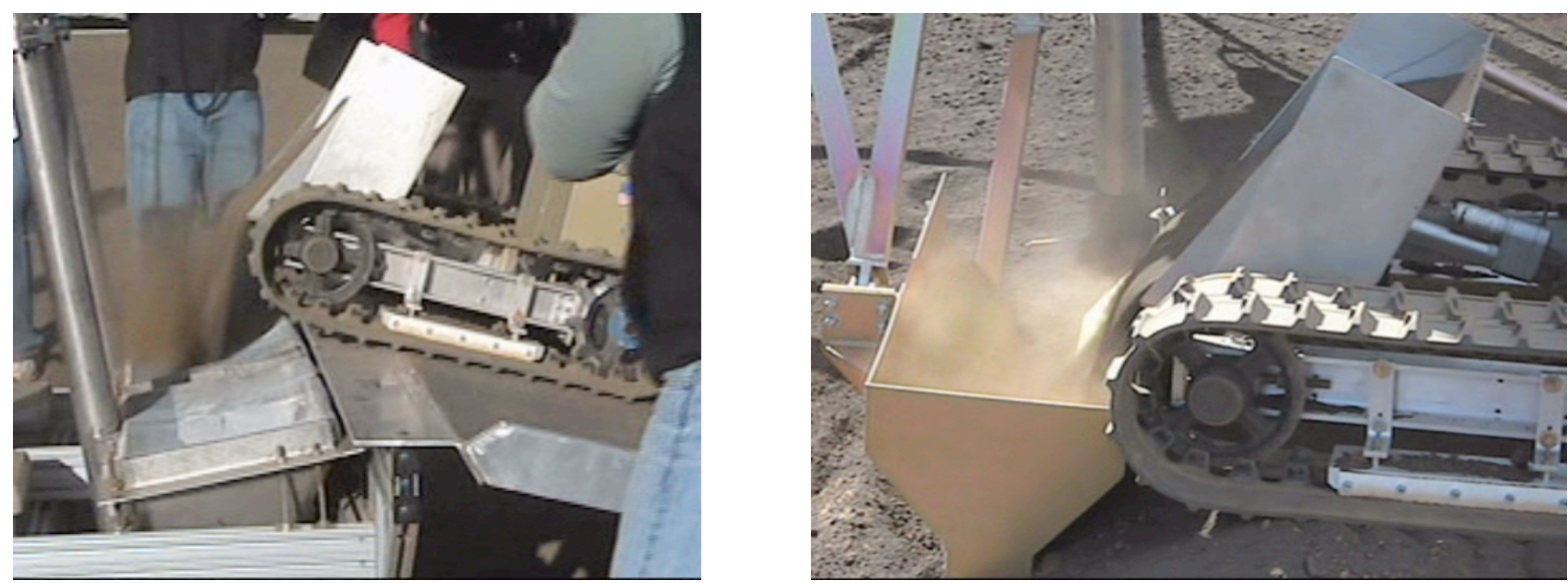

Figure 3. Prototype small excavator delivering $25 \mathrm{~kg}$ load of regolith by driving up a metal ramp (left) and driving on a self-made soil ramp (right). 
digging. However, since the higher-strength leading edge material of the scoop is the component that will apply the digging forces to the soil, the narrower scoop will be slightly lighter in mass. The scoop width must also be matched to the top of the hoppers such that the regolith can be dumped into the hopper without significant regolith spillage. The current assumption is that the excavation site will be up to $100 \mathrm{~m}$ away from the ISRU plant, and the dump-site will be $100 \mathrm{~m}$ away from the ISRU plant and the excavation site, forming three sides of a triangle. With these assumptions, a velocity of $0.5 \mathrm{~m} / \mathrm{s}$, and 10 minutes at the plant to maneuver and dump into the hoppers, the large excavator requires less than 1 hour per day to deliver its single load of $200 \mathrm{~kg}$ of regolith.

For a small excavator, we have assumed a maximum load of $25 \mathrm{~kg}$ of regolith per trip. This will require 8 trips per day to the ISRU plant. Assuming the same distances between excavation site, dump site, and plant, and a velocity of $0.20 \mathrm{~m} / \mathrm{s}$, the small excavator will require approximately $4.5 \mathrm{hrs}$ to deliver its 8 loads to the ISRU plant. Figure 3 shows a prototype of a small excavator ${ }^{9}$ delivering a load of regolith to two different ISRU plant prototypes. In the photo on the left the vehicle is driving up a metal ramp to dump into the hopper; in the photo on the right it is driving up a small ramp that it made from the regolith at the site.

\section{Hydrogen Reduction Reactor}

The hydrogen reduction reaction involves heating the regolith to $800-1100$ degrees $\mathrm{C}$ and reacting with gaseous hydrogen to extract oxygen from the iron-bearing minerals in the form of water. This water is then electrolyzed with the oxygen being stored (either as a liquid or high-pressure gas) and the hydrogen being recycled back into the reactor. Some current concepts for mixing the hydrogen and the regolith are to use a fluidized bed or to use mechanical means to stir the regolith. The mixing causes the hydrogen gas to be well-dispersed and heat to be uniformly distributed. A model has been developed to analyze the physical, thermal, and chemical characteristics of these reactors during operation. ${ }^{10,11}$

One of the first studies performed with the ISRU System Model tool indicated large electrolyzer mass and power requirements when assuming a single reactor operation. This was caused by the non-continuous flow of water from the reactor to the electrolyzer, as a single reactor would only produce water while hydrogen was flowing but not during the times when the reactor was being dumped of spent regolith, filled with fresh regolith, and heated up to operating temperature. The system model showed a clear advantage to using a parallel reactor system, where one reactor is dumping, filling, and heating up while the other is operating. This greatly reduced the mass and power requirements of the electrolyzer and oxygen liquefaction systems, and reduced the heating power requirement as the heating could now also occur nearly continuously. This sensitivity result has been discussed in detail in Refs. 2 and 3. Based on the significant benefits of the parallel reactor concept for hydrogen reduction, two reactors were assumed for all of the analysis in this section.

\section{A. Conversion Time and Energy}

Previous analysis of a hydrogen reduction system assumed that all of the oxygen from the iron-bearing minerals could be extracted in the time available for each batch. The new version of the model now calculates reaction time for any desired yield (conversion), and preliminary validation using tests with JSC-1A simulant has been used to

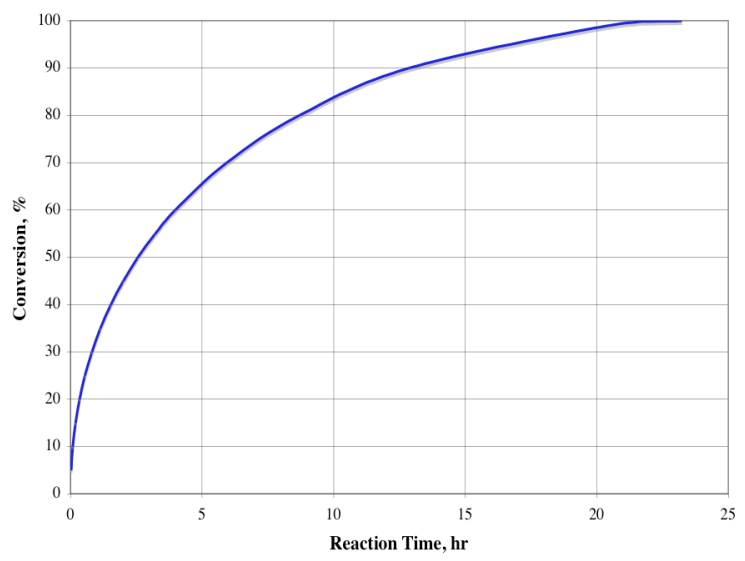

Figure 4. Conversion with time for hydrogen reduction reactor $(10 \mathrm{~kg}$ regolith, $0.2 \mathrm{~m}$ diameter reactor).

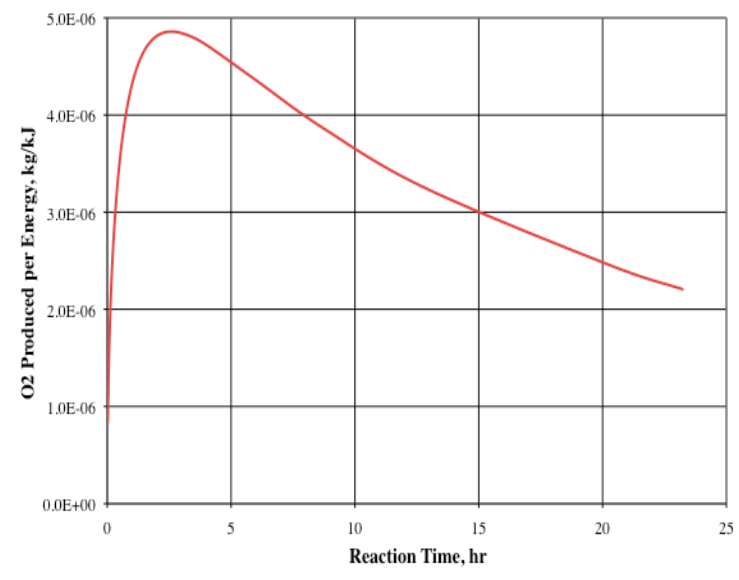

Figure 5. Oxygen produced per energy input (10 $\mathrm{kg}$ regolith, $0.2 \mathrm{~m}$ diameter reactor). 
anchor the algorithms. ${ }^{11}$ Initially, the iron-bearing minerals on the outside of the particles are reacted, and the conversion during this time is mainly limited by the availability of hydrogen which is determined by its flowrate. However, once the outer surfaces have been reacted, additional oxygen can only be extracted if sufficient time is available for the hydrogen gas to diffuse into the particles and the resulting water to diffuse out of the particles. Figure 4 shows a typical conversion curve for reacting $10 \mathrm{~kg}$ of regolith in a $0.2 \mathrm{~m}$ diameter reactor.

From Fig. 4 it is clear that the conversion rate slows down with increasing time, but it is not clear how to decide when to stop the batch and bring in fresh regolith. By understanding the energy required for reaction some insights into optimum processing time can be made. For each batch processed, there is an initial energy investment to heat up the regolith from the lunar surface temperature (assumed to be 0 degrees $\mathrm{C}$ here) to the operating temperature (assumed to be 1000 degrees C). This energy is based on the amount of regolith in the reactor $(10 \mathrm{~kg})$, the time available for heating $(0.9 \mathrm{hr})$, the reactor diameter $(0.2 \mathrm{~m})$, and the insulation properties such as thickness (assumed to be $0.02 \mathrm{~m}$ ). Next, there is a radiative heat loss during processing that is a function of the insulation thickness, reactor diameter, and processing time. As processing time increases and conversion decreases, there is a time at which the amount of oxygen being claimed per energy investment starts to decline. A typical curve showing this relationship is shown in Fig. 5. For this analysis, we assumed no reaction during the heat-up process; future updates to the model will include reaction during heat-up but should not greatly change the shape of this curve.

By combining these two curves (Fig. 6) we can see at what percent conversions the reaction should be stopped in favor of switching to fresh regolith. For these conditions, the most energy efficient operation would be to stop the conversion around $55-65$ percent. Note, however, that the selection of conversion percent affects the amount of regolith that the excavation system needs to deliver, the size of the regolith hoppers, and the size of the reactor. So in this example, stopping the reaction at 60 percent conversion causes the per day regolith to increase from $200 \mathrm{~kg}$ (minimum regolith for $100 \%$ conversion) to $333 \mathrm{~kg}$ per day. For the small dedicated excavator discussed previously, this would increase its workload from 8 deliveries to 13 or 14 deliveries per day, and increase its operations from 4.7 hours to around 8 hours per day. The regolith hoppers would also increase in size or require more frequent refills each day.

\section{B. Batch Size}

In the previous section investigating conversion with processing time, the amount of regolith and the heat-up time were held constant to simplify the parametrics. However, each of those combinations of conversion amount and processing time would produce different amounts of oxygen on a yearly basis. A more interesting analysis is to determine the relationship between number of batches per day, conversion time, batch size, and processing energy for a given amount of annual oxygen production.

For this analysis, we vary the number of batches run per day and that sets the available processing time per day. For example, 12 batches per day allows for 2 hours of processing time per batch. Since we have 2 parallel reactors, the heat-up time was set at 90 percent of the processing time, with the remaining time assumed to be used for dumping and filling the reactor. The conversion percent is then varied until the required processing time equals the time available for that

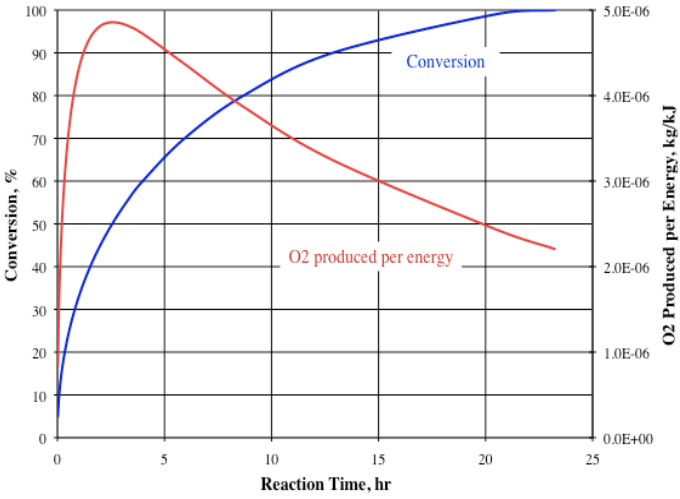

Figure 6. Sensitivity between energy input and conversion fraction $(10 \mathrm{~kg}$ regolith, $0.2 \mathrm{~m}$ diameter reactor). number of batches. Reactor diameter was held constant at $0.4 \mathrm{~m}$ and the yearly oxygen production rate was set at 
$500 \mathrm{~kg}$. Figure 7 shows the results for conversion percent and regolith mass as a function of the number of batches per day. As the number of batches per day decreases, the available time for reaction increases, allowing more time for the diffusion limited extraction of oxygen from the inside of the particles. The amount of regolith processed per batch also increases which increases the size of the reactors.

Figure 8 shows the relationship between the reactor mass, the regolith required per year, and the energy per day for varying batches. While the regolith per batch increased with decreasing batches per day in Fig. 7, the total regolith required per year decreases as the conversion percent increased. Similarly, as the number of batches decreases allowing more time for conversion, we move into the more energy efficient region seen in Figs. 5 and 6, and therefore the total energy per day decreases.

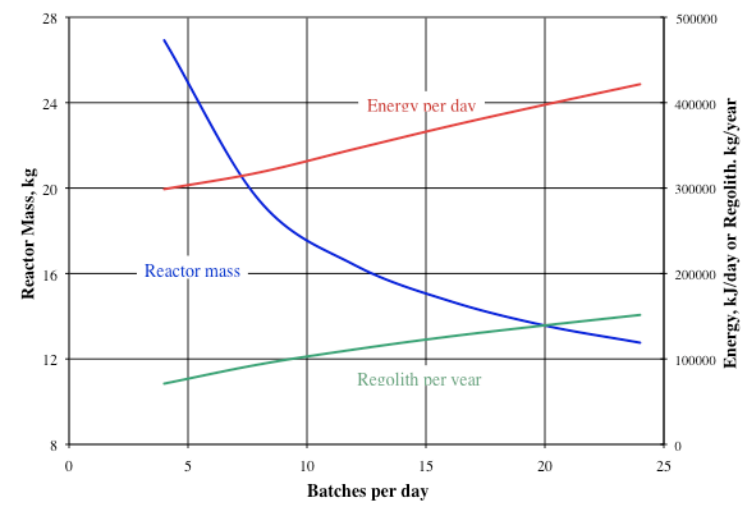

Figure 8. Effect of batches per day on reactor mass, energy, and regolith.

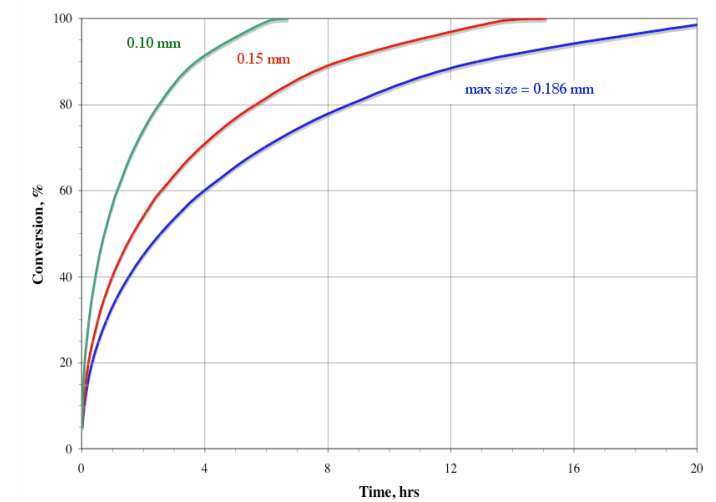

Figure 9. Particle size effects on conversion time.

\section{Particle Size}

For the previous analysis, the maximum particle size was held constant at 186 microns, which provides an average particle size typical of the loose highland regolith based on lunar samples from Apollo. ${ }^{12}$ However, because the later stages of a batch reaction is diffusion limited, it may be beneficial to sort the regolith prior to putting it into the reactor in order to limit the maximum particle size and thereby reduce the diffusion-limited portion of the reaction. Figure 9 shows how the conversion percent as a function of time greatly improves as the maximum particle size is decreased from 186 microns to 100 microns assuming the iron oxide concentration remains constant. There are several concepts being evaluated for size sorting both within NASA and in industry. Once some of these concepts are tested, the mass, energy, and logistics of the size sorting process will need to be included in the sensitivity study to determine if it is worth the increase in yield.

\section{Carbothermal Reduction Reaction}

The carbothermal reduction reaction involves heating the regolith to $1130-1400+$ degrees $C$ to melt the regolith, reacting with methane to deposit carbon onto the melt surface, and extracting oxygen from the minerals in the form of carbon monoxide. The carbon monoxide is then reacted with hydrogen in a second reactor to form methane and water. The water is electrolyzed as in the hydrogen reduction process, with the oxygen being stored and the hydrogen being recycled back into the second reactor. The methane from the second reactor is recycled back to the carbothermal reactor and provides the source of the carbon for the reduction process. Because of the very high temperatures required, early research for the carbothermal process had difficulties with the methane cracking before reaching the reactor and clogging up flow passages. ${ }^{4}$ Also, it was difficult to find reactor vessels that could hold the molten regolith, especially through repeated cycles. More recent research has focused on heating only pockets of the regolith in a bed to the melting temperature and using the remaining regolith bed as an insulating material. ${ }^{13} \mathrm{~A}$ model has been developed to analyze the physical, thermal, and chemical characteristics of this carbothermal reactor concept during operation. ${ }^{14,15}$

The carbothermal process has the potential to extract oxygen from the silicon oxides, iron oxides, and titanium oxides. The total oxide mass in these components is $25-30$ percent of the regolith mass, varying only slightly with location. We have used 25 percent as our maximum oxygen potential in the analyses to follow. Because the carbothermal reactor design being evaluated is more complicated than the hydrogen reduction reactors considered 
above, we are assuming for now a single reactor in these analyses (the electrolyzer requirements can still be managed through the use of a buffer tank for the water).

\section{A. Methane Flow Rate}

The carbothermal reduction system being investigated has a few more parameters to be considered than the hydrogen reduction system. The first parameter that can affect operation is the amount of carbon available for the reaction, and this is controlled by the methane flow rate. Figure 10 shows how the conversion time decreases with increasing methane flow rate for 12 batches per day, $500 \mathrm{~kg}$ of oxygen per year, and a 50 percent conversion. Also shown in the figure is total batch time with the difference between total time and conversion time being the time needed for the molten regolith zones to cool to solid temperature, be removed from the reactor, and fresh regolith brought in for the next batch.

It can also be seen from Fig. 10 that there is a minimum conversion time that can be achieved for a given set of conditions.

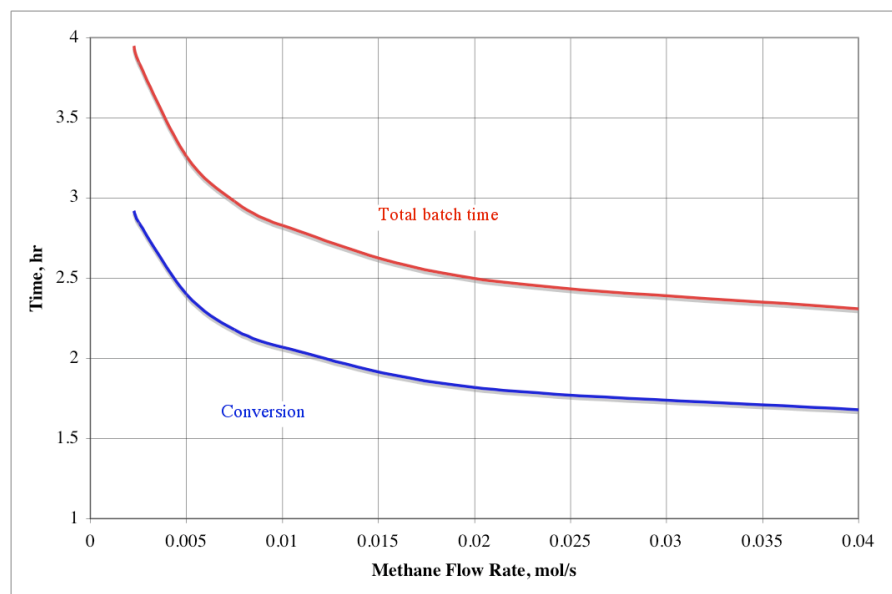

Figure 10. Effect of methane flow rate on conversion time for carbothermal reduction (12 batches per day, $500 \mathrm{~kg} 02$ per year, 50 percent conversion per batch).

Further increasing methane flow rate does not provide any faster conversion. For the particular set of parameters run here a solution does not exist, as 12 batches per day leaves 2 hours total time per batch, which is not achievable under this set of conditions. For all the remaining parametrics that will be shown for the carbothermal reactor, a high methane flow rate will be used such that we are near the minimum conversion time for each set of conditions studied.

\section{B. Conversion Time, Melt Zones, and Energy}

Thermal analysis predicts, and experiments confirm, that each melt zone will continue to grow during the entire processing time. Therefore, for a fixed amount of oxygen production, there is an inverse relation between the conversion time and the number of melt zones. In addition, for a given amount of oxygen desired per batch, fewer melt zones will mean more regolith melted per zone. Figure 11 shows this relationship between conversion time and conversion percent and number of melt zones for processing of $1.5 \mathrm{~kg}$ of regolith per batch. There will also be a

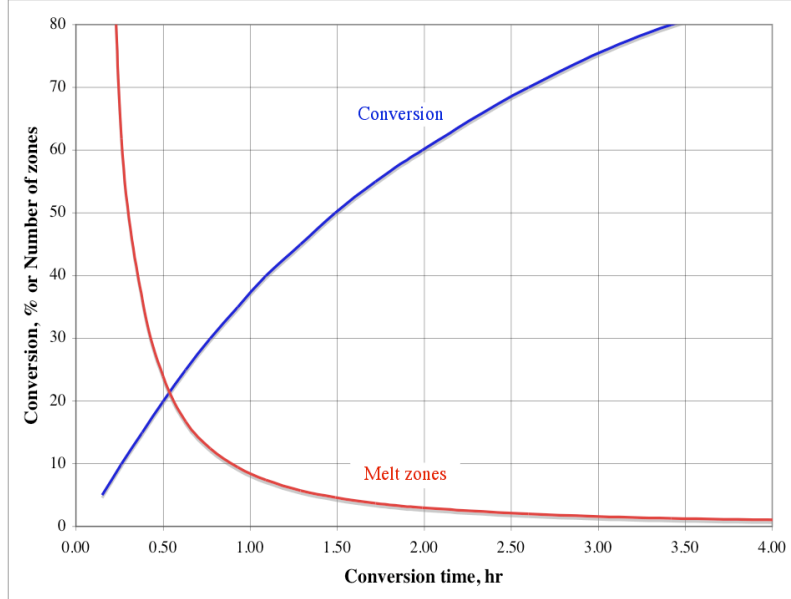

Figure 11. Relationship between conversion time, conversion fraction, and number of melt zones $(1.5$ kg regolith processed).

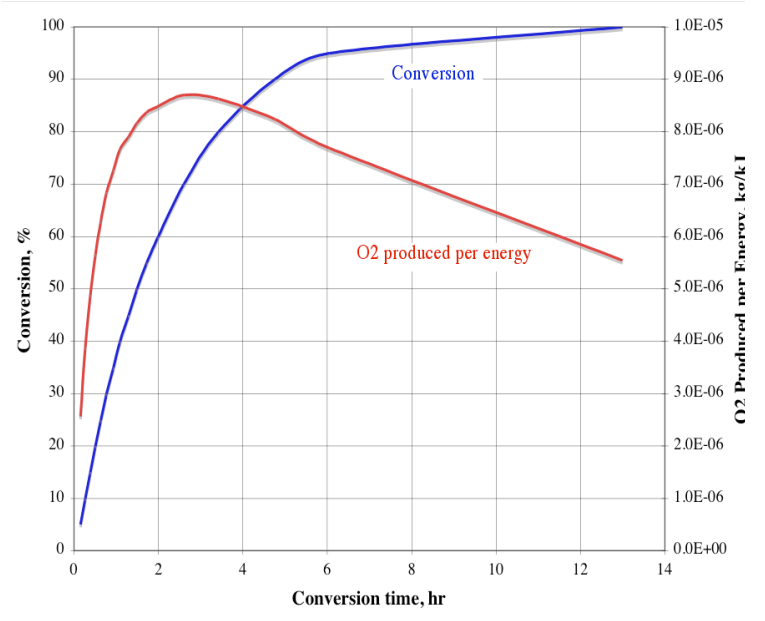

Figure 12. Sensitivity between energy input and conversion fraction ( $1.5 \mathrm{~kg}$ regolith processed). 
maximum conversion time and conversion percent for a given regolith mass to be processed in a batch, as the actual number of melt zones cannot fall below one. For the set of conditions shown in Fig. 11, the maximum conversion time is $4.6 \mathrm{hr}$ for an 86 percent conversion.

As with the hydrogen reduction reactors, there is a relationship between conversion time, conversion percent, and energy used per kilogram of oxygen produced. The reaction rate is dependent on the concentration of the oxide being reduced remaining in the melt, so as oxygen is extracted and the concentration goes down, the reaction rate decreases. As with the hydrogen reduction reaction, at some point there will be a reduction in product extracted for energy invested. Figure 12 shows that the peak of the energy curve is fairly flat between a conversion time of $2-4$ hrs, so there is some flexibility here in considering other factors when selecting the optimum operating conditions.

\section{Batch Size}

As discussed previously, as the number of batches per day decreases the time available for conversion increases, allowing for larger melts for a given regolith processed per batch. But as the number of batches decreases the amount of regolith that must be processed per batch increases, so regolith per batch is not a constant. The amount of regolith processed per batch also depends on the conversion fraction achieved per batch, which is a function of time available. Figures 13 - 15 capture some of these sensitivities. In Fig. 13, the conversion fraction and regolith per

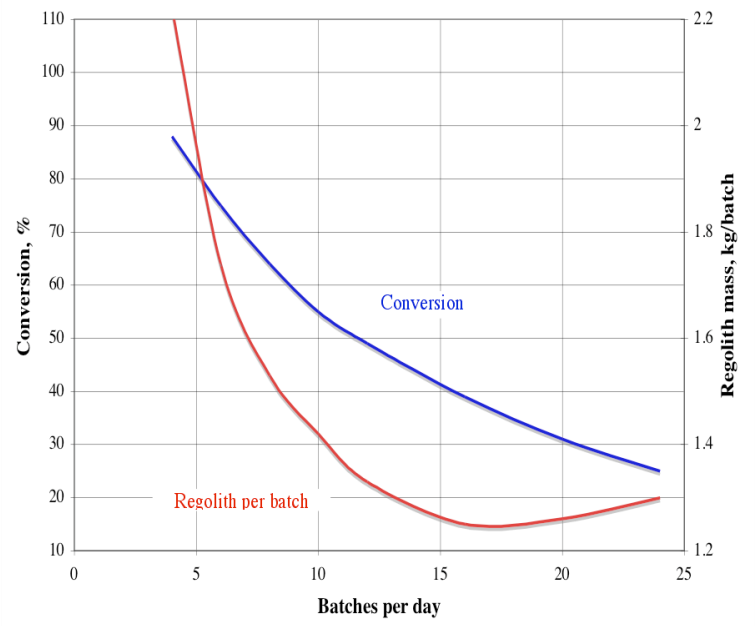

Figure 13. Conversion fraction and regolith required per batch sensitivity to batches per day (500 kg O2 per year).

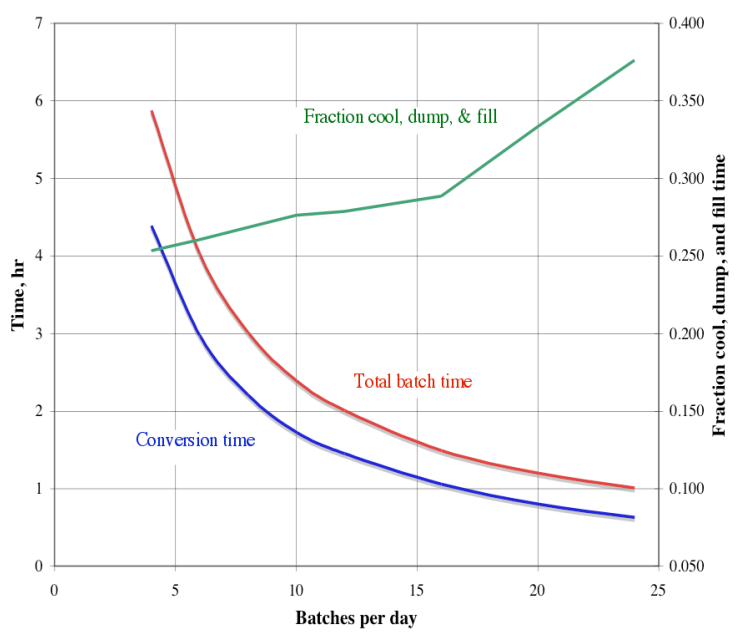

Figure 14. Increasing time spent on cooling, dumping, and filling instead of chemical reaction (500 kg O2 per year).

batch are shown as a function of batches per day. Similar to the hydrogen reduction relation, the conversion fraction decreases with increasing number of batches, as there is less time to let the reaction move towards completion. Regolith per batch also decreases with increasing batch size, although this is a function of both the higher number of batches and the decreasing conversion fraction. For this set of conditions the regolith per batch reaches a minimum around 16 batches per day and then increases.

Because a single reactor was assumed for the carbothermal reduction system, batch time must now include more than just conversion time. For this reactor, batch time also includes time to cool the melts to solid temperature, time to remove the solid processed zones, and time to bring in fresh regolith. As the amount of regolith processed per batch decreases and the number of zones decrease, the dump and fill times begin to reach a plateau. For example, close-packed hexagonal spacing assumed here for the melt zones tends to minimize to 3 rows of melts. For the reactor design described in Ref. 13, the solidified melts are removed one row at a time, such that the removal time will remain constant once the minimum number of rows has been reached. Also, it was assumed in this analysis that once fresh regolith is pushed into the bed, two passes of a smoothing rake will be made to prepare the surface for the next reaction. Figure 14 shows how the fraction of total batch time used for cooling, dumping, and filling begins to rise sharply once these minimum operation times are reached and any further reduction in batch time must all come from available conversion time. 
Figure 15 shows the regolith required per year and the energy per day for the carbothermal reactor. Both of these key performance parameters decrease significantly with decreasing batches per day. The energy reaches a minimum around 6 batches per day, which equates to a conversion time of approximately $3 \mathrm{hrs}$ and a conversion fraction for these conditions of approximately 75 percent. The 3-hour conversion time corresponds to the peak of the oxygen produced per energy input curve examined in Fig. 12.

\section{Conclusions}

Some of the key components of an ISRU system were evaluated to understand how various design parameters affect the performance of a given component and how design decisions for one component may affect the performance of other components in the system. For excavation, both large and small excavation vehicles were examined, with both having potential benefits. While the large excavator can deliver one-day's regolith requirement to the ISRU plant in less than one hour and

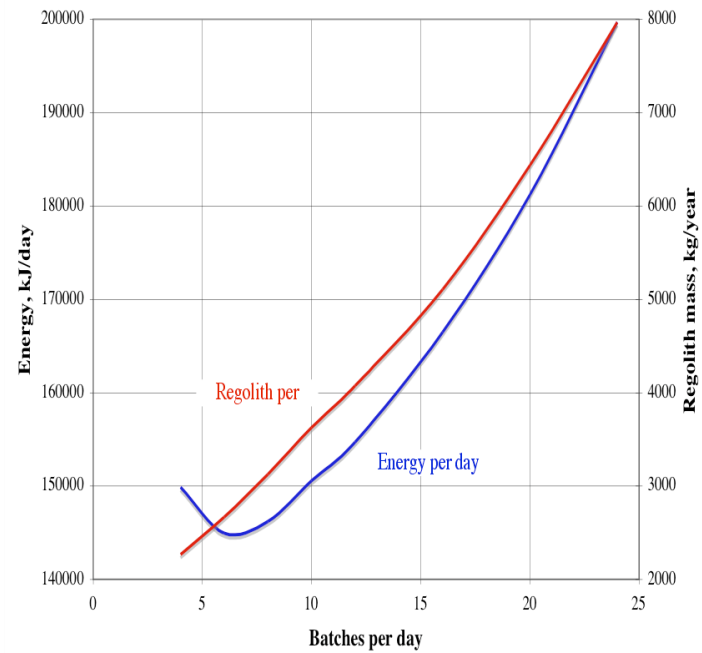

Figure 15. Regolith per year and energy per day sensitivity to batches per day $(500 \mathrm{~kg} O 2$ per year). the small excavator can deliver the regolith in 4.5 hours, more work is needed in the analytical model to validate estimates for mass and power such that a more quantitative comparison can be completed.

The hydrogen reduction reactor was analyzed to understand batch size and conversion fraction effects on reactor mass, reactor energy, and the amount of regolith required per day. The results indicate that by processing larger regolith quantities in fewer batches per day significantly decreases the energy requirement while requiring a modest increase in reactor mass. However, this increase in reactor mass can be partially offset by a reduction in hopper mass, since the larger batch will provide a higher oxygen yield from the regolith resulting in less regolith to be excavated and stored in the hoppers. A reduction in regolith requirement will also reduce the operating time of the small excavators.

The carbothermal reduction reactor was analyzed to understand the relationship between the methane flow rate, batch size, conversion fraction, number of melt zones, and reactor energy. Again, processing larger regolith quantities in fewer batches per day results in a lower energy requirement and in less regolith required per day.

\section{Acknowledgments}

The authors gratefully acknowledge the support of this work by the In-Situ Resource Utilization Project which is a part of the NASA Exploration Technology Development Program.

\section{References}

${ }^{1}$ Sanders, G. B., Larson, W. E., Sacksteder, K. R., and Mclemore, C., "NASA In-Situ Resource Utilization (ISRU) ProjectDevelopment \& Implementation," AIAA Paper No. 2008-7853, AIAA SPACE 2008 Conference \& Exposition, San Diego, California, September 2008.

${ }^{2}$ Larson, W. E., Sanders, G. B., Sacksteder, K. R., Simon, T. M., and Linne, D. L., "NASA's In-Situ Resource Utilization Project: A Path to Sustainable Exploration," IAC-08-A3.2.B13, International Astronautical Congress, Glasgow, Scotland, October 2008.

${ }^{3}$ Sanders, G. B., Larson, W. E., Sacksteder, K. R., Simon, T. M., and Linne, D. L., "Open Architecture for Lunar Surface Systems-Challenges \& Opportunities In Design, Integration, And Partnerships," IAC-08-D3.2.1, International Astronautical Congress, Glasgow, Scotland, October 2008.

${ }^{4}$ Rosenberg, S.D., Beegle, R.L., Jr., Miller, F.E., and Rothenberg, M., "The Manufacture of Propellants for the Support of Advanced Lunar Bases," paper 650835, presented at the Society of Automotive Engineers' National Aeronautic and Space Engineering and Manufacturing Meeting, October 1965.

${ }^{5}$ Steffen, C.J., Freeh, J.E., Linne, D.L., Faykus, E.W., Gallo, C.A., and Green, R.D., "System Modeling of Lunar Oxygen Production: Mass and Power Requirements," paper 2049, in Proceedings of Space Nuclear Conference 2007, June 2007.

${ }^{6}$ Linne, D.L., Santiago-Maldonado, E., Gallo, C.A., Freeh, J.E., Notardonato, W.U., and Mueller, R.P., "ISRU System Study Preliminary Results," in Proceedings of $4^{\text {th }}$ Planetary \& Terrestrial Mining Sciences Symposium, Sudbury, Ontario, Canada, June 2007. 
${ }^{7}$ Wilkinson, R. A and DeGennaro, A., Digging and Pushing Lunar Regolith: Classical Soil Mechanics and the Forces Needed for Excavation and Traction, Journal of Terramechanics, 44(2), 2007.

${ }^{8}$ Gallo, C.A., Wilkinson, R.A., Mueller, R.P., Schuler, J.M., and Nick, A.J., "Comparison of ISRU Excavation System Model Blade Force Methodology and Experimental Results,” AIAA-2009-1387, January 2009.

${ }^{9}$ Caruso, J.J., Spina, D.C., Greer, L.C., Wentworth, T.J., Michele, C., Krasowski, M.J., and Prokop, N.F., "Excavation on the Moon: Regolith Collection for Oxygen Production and Outpost Site Preparation," AIAA-2008-808, January, 2008.

${ }^{10}$ Hegde, U., Balasubramaniam, R., and Gokoglu, S., "Analysis of Thermal and Reaction Times for Hydrogen Reduction of Lunar Regolith," in proceedings of Space Technology and Applications International Forum (STAIF-2008), edited by M. ElGenk, AIP Conference Proceedings 504, New York, 2008.

${ }^{11}$ Hegde, U., Balasubramaniam, R., and Gokoglu, S., "Development and Validation of a Model for Hydrogen Reduction of JSC-1A," AIAA-2009-1389, January 2009.

${ }^{12}$ Heiken, G. H., Lunar Sourcebook, Cambridge University Press, NY, 1991.

${ }^{13}$ ORBITEC, "Carbothermal Reduction of Lunar Regolith," Phase I Final Report OTC-GS0167-FR-06-1, Orbital Technologies Corporation (ORBITEC), Madison, Wisconsin, USA, September 2006.

${ }^{14}$ Balasubramaniam, R., Hegde, U., and Gokoglu, S., "Carbothermal Processing of Lunar Regolith Using Methane," in proceedings of Space Technology and Applications International Forum (STAIF-2008), edited by M. El-Genk, AIP Conference Proceedings 504, New York, 2008.

${ }^{15}$ Balasubramaniam, R., Gokoglu, S., and Hegde, U., "Determination of Chemical Kinetic Rate Constants of a Model for Carbothermal Processing of Lunar Regolith Simulant Using Methane,” AIAA-2009-1390, January 2009. 